Ophthalmologe 2013 · 110:497

DOI 10.1007/s00347-013-2870-6

Online publiziert: 14. Juni 2013

(c) Springer-Verlag Berlin Heidelberg 2013

F.G. Holz

Universitäts-Augenklinik, Bonn

\title{
Update Ophthalmologie
}

\section{Kernthemen prägnant zusammengefasst und kritisch bewertet}

\section{Liebe Leserinnen, liebe Leser,}

das Angebot an Publikationen im wissenschaftlichen Raum nimmt stetig zu, sodass man meist nur durch zeitintensive Recherchen die gewünschten Informationen findet. Um Sie in dieser Informationsflut zu unterstützen und die für Sie entscheidenden Informationen herauszufiltern, haben wir die neue Rubrik „Update Ophthalmologie“ ins Leben gerufen. Getragen von dem Weiterbildungsgedanken der Zeitschrift, fasst diese Rubrik für die jeweiligen Themenbereiche einige der wichtigsten im letzten Jahr in nationalen und internationalen wissenschaftlichen Zeitschriften publizierten Ergebnisse klinischer Studien zusammen. Die Studienergebnisse werden zudem von renommierten Experten bewertet, die zusätzlich konkrete Empfehlungen für die Umsetzung in die Praxis an die Hand geben.

In dieser Ausgabe starten wir mit einem Beitrag von Prof. Dr. Claus Cursiefen zum trockenen Auge. Er beleuchtet darin u.a. neue Erkenntnisse zur Rolle der Ganzkörperhydrierung für die Tränenfilmosmolarität, wobei eine suboptimale Hydrierung ein trockenes Auge begünstigen kann. Weiterhin gibt es auch neue Erkenntnisse der Rolle von Pterygien hinsichtlich der Tränenosmolarität. Hinsichtlich therapeutischer Ansätze geht er auf die Rolle von Eigenserumaugentropfen bei besonders schweren Ausprägungsformen des Sicca-Syndroms ein wie auch auf die Amnionmembrantransplantation in Gegenwart persistierender Hornhautepitheldefekte. Neue Arbeiten weisen darauf hin, dass die Sondierung der MeibomDrüse bei obstruktiver Form der Mei-
bom-Drüsen-Dysfunktion wirksam sein kann. Dargestellt wird auch eine Arbeit, die den fortschreitenden Verlust von Meibom-Drüsen unter langjähriger lokaler Antiglaukomatosa-Therapie aufzeigt.

Mit dieser Zusammenstellung von aktuellen Publikationen zum trockenen Auge hoffen wir, auch für Sie die interessantesten Aspekte in diesem sich rasant entwickelnden Bereich der Ophthalmologie herausgefiltert zu haben. Wir sind überzeugt, dass diese Rubrik ein Gewinn für die Zeitschrift ist und einen wichtigen Beitrag zur wissenschaftlichen Diskussionen in Der Ophthalmologe liefert. Allen beteiligten Autoren danke ich für Ihre wertvollen Beiträge. Unseren Lesern wünsche ich eine spannende und anregende Lektüre.

Ihr

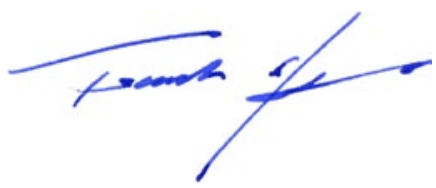

Prof. Dr. Frank Holz

\section{Korrespondenzadresse}

\section{Prof. Dr. F. Holz}

Universitäts-Augenklinik Bonn

Ernst-Abbe-Str. 2

53127 Bonn

frank.holz@ukb.uni-bonn.de 